\title{
INTERPRETIVE SUMMARIES, JUNE 2011
}

Effects of milk composition, stir-out time, and pressing duration on curd moisture and yield. By Everard et al., page 2673. Cheese curd, produced from milk of varying protein level and protein:fat ratio, was drained at various times during syneresis and monitored for moisture and yield over a 22-h pressing period. Curd moisture after pressing decreased with longer stir-out time and pressing duration, and the latter experimental variables had an interactive effect on curd moisture and yield. These results have implications for cheese yield, efficiency of production, and ripening, because moisture control is important for consistent ripening. Control of stir-out and pressing duration would facilitate increased control of final cheese quality, because cheese moisture and associated lactose content influence flavor and texture, indicating a role for process analytic technology in minimizing variation in cheese characteristics.

Factors associated with coliform count in unpasteurized bulk milk. By Pantoja et al., page 2680. Coliform bacteria present in farm milk can be hazardous to human health (especially when unpasteurized milk is consumed) and detrimental for the quality of milk and dairy products. Ten Wisconsin dairies were visited weekly over 10 wk to identify factors associated with coliform count in bulk milk. Hygiene-related factors such as the occurrence of milking machine wash failures, coliform contamination of liners, and rate of milking unit falling to the parlor's platform were associated with bulk milk coliform count and suggest that managing and monitoring these practices have potential for minimizing milk coliform contamination.

Standardization of milk using cold ultrafiltration for the manufacture of Swiss cheese: Effect of altering coagulation conditions on yield and cheese quality. By Govindasamy-Lucey et al., page 2719. In recent years, interest has been increasing in concentrating milk by membrane filtration at low temperature (to maintain microbial quality). This study shows that the use of these cold concentrated milks improved cheese yield with no significant effect on ripening or sensory quality. The faster coagulation and firming caused by increased protein can be reduced by altering the renneting conditions. A cheese yield prediction equation was developed that successfully predicted yield. These concentrates are beneficial to cheese plants that want to increase productivity without negatively affecting product quality.

Zinc-binding capacity of yak casein hydrolysate and the zinc-releasing characteristics of casein hydrolysate-zinc complexes. By Wang et al., page 2731. Zinc deficiency is prevalent in populations that consume mainly plant-based food. It can cause many organ dysfunctions and lead to complications associated with central nervous system, immune system, and reproductive system function. Yak casein hydrolysate can bind with zinc and improve its solubility and dialyzability under a simulated gastrointestinal environment. Yak casein hydrolysate-zinc complexes may have potential as effective zinc delivery vehicles to enhance zinc status.

Goat milk during iron repletion improves bone turnover impaired by severe iron deficiency. $B y$ Diaz-Castro et al., page 2752. Milk has high Ca bioavailability and can positively affect the process of bone mineralization; however, Fe deficiency impairs the bone remodeling. Goat milk increases the parameters of bone formation in anemic rats only $10 \mathrm{~d}$ after supplying this milk. If the supply of goat or cow milk-based diets is prolonged $(30 \mathrm{~d})$, biomarkers of bone formation and resorption are similar in controls and anemic rats. Therefore, bone demineralization induced by Fe-deficiency anemia is recovered, but occurs faster with goat milk.

Proteolysis of milk fat globule membrane proteins during in vitro gastric digestion of milk. By Ye et al., page 2762. In its natural state, milk is a classical oil-in-water emulsion in which the fat globules are stabilized with milk fat globule membrane (MFGM). The physicochemical behavior of fat droplets in gastric fluid and intestinal fluid is an area of interest, in particular, their morphology during gastric and intestinal digestion. Changes in the physicochemical properties of fat globules and dispersed hydrolytic products are important in understanding the digestion and absorption of lipids in milk products. This study attempted to understand the physicochemical characteristics of fat globules and role of MFGM in the digestion of milk fat. This paper provides useful information on the effect of the gastric environment and the presence of proteolytic enzymes on milk fat globules and the MFGM.

Short communication: Proteins in heat-processed skim milk powder have no positive effects on bone loss of ovariectomized rats. $B y D u$ et al., page 2771. Proteins in heat-processed skimmed milk powder (0.04 to $0.40 \mathrm{~g} / \mathrm{d}$ ) have no positive effects on bone loss in ovariectomized rats, probably because of the thermal denaturation of proteins in the processing of bovine milk.

Short communication: Occurrence of aflatoxin M1 in the Manchego cheese supply chain. By Rubio et al., page 2775. Manchego cheese is a Protected Designation of Origin product that must guarantee high quality and food safety for consumers; as such, strict 
controls are in place from the milk to the farm and to the final manufactured dairy product. Aflatoxin M1 contamination can occur along the chain and become a health problem due to its carcinogenic properties. Screening of this toxin has been carried out on different farms and in cheese factories, with around $99 \%$ of the analyzed samples having afltoxin M1 levels below the European Union limit. Therefore, dairy products manufactured with Manchega ewe milk impose no risk in relation to aflatoxin M1.

First evidence of the presence of genomic islands in Escherichia coli P4, a mammary pathogen frequently used to induce experimental mastitis. By Dufour et al., page 2779. Pathogenicity of strains of Escherichia coli that provoke bovine mastitis remains poorly understood despite the economic losses they cause in dairy cattle. Identification of specific genes that could be involved in pathogenicity of the mammary pathogen strains of E. coli (and that are frequently clustered into genomic islands in other strains of E. coli) is important for the understanding of this disease. This paper describes the phylogenetic study and the first discovery of genomic islands in the strain E. coli $\mathrm{P} 4$, which has been isolated from a severe bovine mastitis and is widely used to induce experimental bovine mastitis.

Luteolytic effects of cloprostenol sodium in lactating dairy cows treated with G6G/Ovsynch. By Martins et al., page 2806. Ovsynch is widely used as a reproductive management tool for dairy farms. Cows treated with cloprostenol sodium had lower concentrations of progesterone during the first $12 \mathrm{~h}$ following induced luteolysis and subsequently greater concentrations of estradiol $48 \mathrm{~h}$ posttreatment compared with cows treated with dinoprost tromethamine.

Effects of cloprostenol sodium at final prostaglandin $F_{2 \alpha}$ of Ovsynch on complete luteolysis and pregnancy per artificial insemination in lactating dairy cows. By Martins et al., page 2815. Fertility of lactating dairy cows has decreased significantly during the past $30 \mathrm{yr}$. This study focused on improving the fertility of lactating dairy cows by controlling ovarian function. Cloprostenol did not enhance luteolysis or conception rates compared with dinoprost. Cows with greater concentrations of progesterone had greater chances for luteolysis and conception. Dairy producers and their veterinarians may utilize these data to improve the reproductive performance and profitability of their herds.

Case-control study: Productivity and longevity of dairy cows that tested positive for infection with Mycobacterium avium ssp. paratuberculosis as heifers compared with age-matched controls.
By Pillars et al., page 2825. A case-control study was conducted to compare the productivity and longevity of cows that had tested positive for Mycobacterium avium ssp. paratuberculosis (MAP, the causative agent of Johne's disease), prior to 24 mo of age, to age-matched herdmates. Cases were individually matched to 4 controls. No significant differences were found between cases and controls in terms of herd longevity; milk, fat, and protein production; or MAP test and clinical status as cows. Thus, testing immature dairy heifers for MAP is not currently economically justifiable.

Rumen $\mathrm{pH}$ and fermentation characteristics in dairy cows supplemented with Megasphaera elsdenii NCIMB 41125 in early lactation. By Aikman et al., page 2840. Dairy cows were dosed with Megasphaera elsdenii to enhance the naturally occurring rumen population of lactate-utilizing bacteria in the critical period of increasing feed intake after calving. Rumen $\mathrm{pH}$ fluctuated less and remained higher longer in cows that received M. elsdenii, and a shift was observed in rumen volatile fatty acid production from acetate towards propionate. This could have beneficial effects on health, energy balance, and milk yield in early lactation cows.

Supranutritional selenium increases mammary gland vascularity in postpartum ewe lambs. $B y$ Vonnahme et al., page 2850. Feeding ewes supranutritional selenium (Se) from breeding through parturition increased capillary vascularity in the mammary gland. Adequate vascularity in the mammary gland is imperative for proper milk production. In a time when nutrients may not be adequate, supplementing Se may help offset the negative effects of poor nutrition on mammary gland development and colostrum output. Ensuring proper colostrum and milk production will increase the survivability of the neonate and promote growth and development during postnatal life.

Effect of trans-10, cis-12 conjugated linoleic acid on performance, adipose depot weights, and liver weight in early-lactation dairy cows. By von Soosten et al., page 2859. Conjugated linoleic acids (CLA) altered nutrient partitioning, reduced adipose depots, and enlarged livers in studies on different mammals. The present study investigated the occurrence of these effects during feeding of CLA in dairy cows. Energy spared by reduced milk energy concentration was repartitioned to higher milk yield. Liver enlargement took place in a physiological range and was not fortified by CLA supplementation. The omental, mesenteric, and subcutaneous adipose depots were not influenced by CLA treatment. The retroperitoneal fat depot underwent the greatest changes in early lactation, and a decelerated mobilization could be suggested within $42 \mathrm{~d}$ in milk after calving. 
Physiological and conjugated linoleic acid-induced changes of adipocyte size in different fat depots of dairy cows during early lactation. By Akter et al., page 2871. Early lactation is accompanied by massive mobilization of body reserves. The concomitant changes of adipocyte size are nonuniform in different subcutaneous and visceral fat depots: visceral (i.e., retroperitoneal) adipocyte sizes were significantly smaller on d 105 than on d 1 of lactation, whereas subcutaneous adipocytes remained unchanged. Supplementation of dairy cows with conjugated linoleic acids (CLA) reportedly decreases milk fat but leaves body fat unaffected; however, the decreased adipocyte sizes that we observed in both subcutaneous and visceral fat depots in CLA-supplemented cows versus the control group indicates lipolytic or antilipogenic effects of CLA.

The effect of lameness on the resting behavior and metabolic status of dairy cattle during the transition period in a freestall-housed dairy herd. By Calderon and Cook, page 2883. Lying time declined during the period immediately before calving. Lame cows had elevated lying times throughout the transition period compared with nonlame cows, and moderately and severely lame cows had a significantly greater number of lying bouts at the point of calving, which may reflect increased hypersensitivity to pain and discomfort associated with lameness and parturition. Lameness was associated with elevated risk for ketosis during the postpartum period.

Measurement of acceleration while walking as an automated method for gait assessment in dairy cattle. By Chapinal et al., page 2895. In 2 experiments, 12 and 24 multiparous cows were fitted with five 3-dimensional accelerometers attached to each leg and the back. Acceleration measures, gait, and walking speed were assessed while cows walked in a straight line on concrete or rubber. Different measures of acceleration were correlated to walking speed, overall gait, and visually assessed asymmetry of the steps, particularly when the accelerometers were attached to the legs. Accelerometers identified differences in acceleration when cows walked on concrete or rubber. Three-dimensional accelerometers seem to be a promising tool for lameness detection on farm and to study walking surfaces.

Estimating test characteristics of somatic cell count to detect Staphylococcus aureus-infected dairy goats using latent class analysis. By Koop et al., page 2902. This study shows that composite somatic cell count has acceptable test properties to detect Staphylococcus aureus-infected dairy goats. This implies that somatic cell count can be used as a screening tool for intramammary infection in dairy goats.
Glucose transporter and hypoxia-associated gene expression in the mammary gland of transition dairy cattle. By Mattmiller et al., page 2912. In the dairy cow, glucose largely serves as the substrate for lactose synthesis during lactation. Research in bovine mammary glucose transporter expression and function has primarily focused on whole tissue and epithelial cells during the transition period. Because glucose must be supplied to the mammary gland from the bloodstream, our study characterized expression and function of these transporters in endothelial cells. Additionally, expression was characterized over the entire lactation cycle and correlated with hypoxia-associated genes that may exert some control over glucose transporters during increased metabolic activity.

Distribution of coagulase-negative Staphylococcus species from milk and environment differs between herds. By Piessens et al., page 2933. Coagulase-negative staphylococci (CNS) are the predominating pathogens causing intramammary infections (IMI) in dairy cows. More research on epidemiology of this diverse group of species is needed to explain this. The cows' environment is thought to be a possible source for CNS IMI and this was investigated in a longitudinal field study on CNS from cowmilk and the environment in 6 dairy herds. Distribution of CNS species varied among herds. Epidemiology of CNS infecting udders differs substantially between species: some had an environmental reservoir, whereas others originated from other sources, such as cow's skin, milker's hands, or other cows.

Does the Milk Income Loss Contract program improve technical efficiency of US dairy farms? By Chang and Mishra, page 2945. Due to volatility in income of dairy farmers, the Milk Income Loss Contract program was introduced in the 2002 farm bill and extended in the 2008 farm bill. This paper addresses a policy issue of this program by empirically assessing whether the program can improve production efficiency of US dairy farms. Results showed that significant effects of the Milk Income Loss Contract payments were only evident among large dairy farms. In contrast, no significant effects were found for small and mediumsized farms.

Risk factors associated with hair loss, ulceration, and swelling at the hock in freestall-housed UK dairy herds. By Potterton et al., page 2952. The risk factors associated with 3 presentations of hock lesions in dairy cattle (hair loss, ulceration, and swelling) were investigated, with 2,982 cows examined across 63 UK herds. Five risk factors were common to hair loss and ulceration and 1 was common to hair loss and swelling, 
with a number unique to each of the lesion presentations. Ulceration may not always be an extension of hair loss, as is implied by most scoring systems. Additionally, the etiology of swelling may differ from that of hair loss and ulceration. Longitudinal work is required to confirm these hypotheses.

Evaluation of portal blood flow using transcutaneous and intraoperative Doppler ultrasonography in dairy cows with fatty liver. By Starke et al., page 2964. Portal blood flow (PBF) was investigated in 80 dairy cows with different values of fatty liver by means of intraoperative Doppler ultrasonography. The venous pulsatility index (VPI) decreased with increasing triacylglycerol content. Velocities of PBF correlated negatively with hepatic triacylglycerol content. The lower VPI and PBF velocities in cows with fatty liver and the negative correlations with the degree of hepatosteatosis may be explained by a decrease of vascular compliance in the liver because of fatty infiltration. These changes were particularly pronounced when hepatic triacylglycerol content exceeded $150 \mathrm{mg} / \mathrm{g}$ of liver fresh weight.

Short communication: Farm and socioeconomic characteristics of the top 100 dairy farm counties in the United States. By Dechow, page 2972. The top 100 counties generated more than half of US dairy sales in 2007. Counties varied widely in farm demographics, with as few as 5 very large dairy farms that averaged $\$ 17,924,000$ in dairy sales per farm to as many as 1,730 dairy farms with less than $\$ 250,000$ in dairy sales per farm. Socioeconomic conditions in the county were more favorably associated with having many dairy farms than having high dairy sales.

Effect of ruminal pulse dose of polyunsaturated fatty acids on ruminal microbial populations and duodenal flow and milk profiles of fatty acids. By Liu et al., page 2977. The objective of this study was to quantify the changes in select ruminal bacterial populations, duodenal flow of fatty acids, and milk fatty acid profile in response to ruminal pulse dose with linoleic acid (LA) and free docosahexaenoic acid (DHA), and the combination thereof. Ruminal pulse dose of DHA increased Megasphaera elsdenii, decreased Fibrobacter succinogenes, but had no effect on Butyrivibrio fibrisolvens or Ruminococcus flavefaciens. Cows receiving pulse dose of LA or DHA showed increases both in flow of vaccenic acid (VA) to duodenum and in proportions of potentially health-promoting fatty acids such as VA and cis-9, trans-11 conjugated linoleic acid in milk fat. The results of this study are useful in formulation of dietary rations to enhance beneficial conjugated linoleic acid content in milk.
Relation of net portal flux of nitrogen compounds with dietary characteristics in ruminants: A meta-analysis approach. By Martineau et al., page 2986. As a first step in improving the efficiency of nitrogen utilization in ruminants, an adequate estimation, from dietary characteristics, of the amounts of nitrogenous compounds absorbed is required. From meta-analyses of published data, net portal flux of amino acids, ammonia, and urea were best related to $\mathrm{N}$ intake, ration concentration of total digestible nutrients, and neutral detergent fiber intake. Better knowledge of dietary factors affecting absorption of amino acids and return of urea into the gut will help to improve balancing rations for ruminants.

Is rumen development in newborn calves affected by different liquid feeds and small intestine development? By Górka et al., page 3002. Justified for economic reasons, early weaning of dairy calves requires fast rumen development. However, widely accepted use of milk replacers instead of whole milk in dairy calf nutrition may slow development of the small intestine and, indirectly, the rumen. The aim of the study was to determine the effect of different liquid feeds on small intestine and rumen development in calves. Feeding calves milk replacer instead of whole milk slowed small intestine and rumen development, whereas dietary supplementation of sodium butyrate in milk replacer accelerated it.

Prospects for using nonconventional feeds in diets for Awassi dairy sheep in Syria. By Hilali et al., page 3014. In the Middle East and other dry areas, available agro-industrial by-products not currently used by dairy sheep farmers can be integrated into animal diets. Using nonconventional feedstuffs can improve traditional feeding methods and reduce feeding costs. Diets formulated with nonconventional feeds and tested on Awassi dairy ewes against unbalanced traditional diets enhanced milk yield and composition and reduced costs at the farm level. Use of these nonconventional diets would increase the livelihoods of resource-poor farmers in the Middle East.

Effects of feeding different levels of dietary fiber through the addition of corn stover on nutrient utilization of dairy heifers precision-fed high and low concentrate diets. By Lascano and Heinrichs, page 3025. Nutrient utilization of dairy heifers fed low or high concentrate diets with different levels of forage fiber through the replacement of corn silage by corn stover inclusion was evaluated. Dry matter digestibility was reduced as dietary fiber increased; fiber digestibility was enhanced quadratically with the highest digestibility at intermediate levels of dietary fiber. Re- 
tention of $\mathrm{N}$ and microbial protein synthesis interacted quadratically between concentrate and fiber levels in the diet. The inclusion of fiber plays an important role and interacts with concentrate levels in the diet when fed precisely to meet dairy heifer requirements.

Effects of including corn distillers dried grains with solubles in dairy calf feeds. By Suarez-Mena et al., page 303\%. Including 39 to $49 \%$ corn distillers dried grains with solubles to replace soybean meal and some corn in diets of calves $<3$ mo of age reduced weight gain 6 to $10 \%$ and decreased dry matter digestibility $10 \%$ in 7 -wk-old calves. Inclusion of $20 \%$ distillers grains in the diet of 2- to 3-mo-old calves reduced weight gain by $4 \%$. Thus, the recommendation from this research is to include $<20 \%$ distillers grains in diets of calves $<3$ mo old and up to $20 \%$ in preweaned calves. Also, in this study feeding milk replacer at 940 versus $630 \mathrm{~g} / \mathrm{d}$ reduced grain intake and limited rumen development.

Corn grain and liquid feed as nonfiber carbohydrate sources in diets for lactating dairy cows. By Eastridge et al., page 3045. Corn processing (steam-flaked versus ground corn), particle size of corn grain (fine versus coarse), and sugar from a liquid feed were studied with lactating cows in mid lactation. The steam-flaked and fine-ground corn were likely more extensively fermented in the rumen, without adversely affecting dry matter intake or neutral detergent fiber digestibility. The liquid feed appeared to be more beneficial with the fine-ground compared with coarseground corn. Feeding finely ground corn increased starch digestibility and provides potential for improved nutrient utilization.

Mustard bran in lactating dairy cow diets. By Maiga et al., page 3054. This study compared feed intake, milk production, and milk components of Holstein cows fed a diet containing $8 \%$ oriental mustard bran versus controls. In experiment 1 , mustard bran replaced a portion of soybean meal and beet pulp in the control diet. In experiment 2 , mustard bran replaced a portion of soybean meal and sunflower seed in the control diet. Feed intake was not changed in experiment 1 but was increased with mustard bran in experiment 2. Mustard bran had a mixed effect on milk production but has no effects on milk components and component yields except milk urea; milk quality was not affected. Feeding $8 \%$ mustard bran presents hemolytic danger to lactating dairy cows.

Evaluation of models to predict the stoichiometry of volatile fatty acid profiles in rumen fluid of lactating Holstein cows. By Morvay et al., page 3063. An accurate prediction of the proportions between individual volatile fatty acids (VFA) in the rumen is of interest because variation in VFA profile is associated with variation in methane production, nutrient partitioning, and milk composition. The aim of this study was to evaluate 6 rumen VFA stoichiometric models for their ability to predict in vivo VFA molar proportions. Predictive performance varied among models. The move toward feed evaluation systems based on animal response rather than animal requirements might necessitate an improved representation of, particularly, propionate and butyrate formation than is provided by current VFA stoichiometry models.

Effect of forage-to-concentrate ratio in dairy cow diets on emission of methane, carbon dioxide and ammonia, lactation performance, and manure excretion. By Aguerre et al., page 3081. Our objective was to determine the effect of feeding lactating dairy cows with diets differing in forage-to-concentrate ratios on production performance and the undesirable atmospheric emission of methane, ammonia, and carbon dioxide. Increasing the proportion of forage in the diet from 47 to $68 \%$ while maintaining dietary crude protein did not affect milk production or emissions of ammonia or carbon dioxide, but increased methane emission per unit of energy-corrected milk by $25 \%$. Methane emission can be mitigated substantially with no effect on the emission of ammonia- $\mathrm{N}$ by formulating diets within the range of existing feeding guidelines and practices.

Dietary inclusion of diallyl disulfide, yucca powder, calcium fumarate, an extruded linseed product, or medium-chain fatty acids does not affect methane production in lactating dairy cows. By van Zijderveld et al., page 3094. Enteric methane production accounts for more than half of all greenhouse gases produced on the dairy farm. In the studies described in this paper, 5 feed components (diallyl disulfide, yucca powder, calcium fumarate, a product containing extruded linseed, and a mixture of capric and caprylic acids) were investigated for their methane-decreasing capacity. No methane reductions were observed when these products were tested in dairy cows. Results of these studies highlight the need to validate in vitro results with in vivo data.

The effect of high-sugar grass on predicted nitrogen excretion and milk yield simulated using a dynamic model. By Ellis et al., page 3105. Highsugar grass varieties have received considerable attention for their ability to reduce nitrogen excretion in cattle. This work took a modelling approach to evaluate and explain the variation in the effect of high-sugar grass on simulated nitrogen excretion and milk yield. Both literature data and analysis of simulation diets including high-sugar grass were considered. The model predicted observed nitrogen and milk yield results well. Variation in nitrogen excretion results could largely be explained by the water-soluble carbohydrate differ- 
ence $(\mathrm{g} / \mathrm{kg}$ of dry matter) between treatments and the changes in crude protein and neutral detergent fiber content of the rest of the plant.

Short communication: Responses to supplemental Saccharomyces cerevisiae fermentation product and triticale grain in dairy cows grazing high-quality pasture in early lactation. By Irvine et al., page 3119. Cows grazing fresh pasture and receiving 3 or $6 \mathrm{~kg}$ of dry matter (DM) of crushed triticale per day were supplemented with 0 or $60 \mathrm{~g}$ of a Saccharomyces cerevisiae fermentation product per cow per day. The $S$. cerevisiae fermentation product increased milk lactose content but did not affect estimated dry matter intake, milk production, colostrum yield or quality, or metabolic indicators of energy status, irrespective of triticale supplementation level. Supplementation with triticale increased milk protein percentage and yield.

Short communication: Limit feeding dairy heifers: Effect of feed bunk space and provision of a low-nutritive feedstuff. By Greter et al., page 3124. The objectives of this study were to examine the behavioral effects of providing limit-fed dairy heifers with increased amounts of feed bunk space (0.34 to $0.68 \mathrm{~m} /$ heifer) and to determine if effects of extra feed bunk space are similar to effects seen when providing a low-nutritive feedstuff alongside a total mixed ration (TMR). Neither increased feed bunk space nor provision of straw will reduce competition for, or slow consumption rates of, a limit-fed TMR. Provision of straw alongside a limit-fed TMR did increase dry matter intake, contributing to further rumen fill and allowing heifers to increase daily feeding time, thereby satisfying natural foraging requirements.

Simultaneous estimation of genotype by environment interaction accounting for discrete and continuous environmental descriptors in Irish dairy cattle. By Windig et al., page 313\%. A model is developed that can simultaneously analyze the effect of both discrete and continuous environmental factors on genetic parameters. Such a model is especially useful in estimating genetic correlations within traits between different production systems and levels. The model was applied to spring-calving and year-round calving production systems in Ireland, and showed that the genetic correlation for somatic cell score between the two systems was lower when average herd milk production was taken into account compared with a model without average herd production.

Whole-genome association study for milk protein composition in dairy cattle. By Schopen et al., page 3148. Genome-wide association was studied using 50,228 single nucleotide polymorphism markers for 1,713 Dutch Holstein-Friesian cows. In total, 31 genomic regions distributed across 20 chromosomes showed significant effects on milk protein content or composition. Main genomic regions associated with milk protein composition or protein percentage were on chromosomes $5,6,11$, and 14 . The associations on chromosomes 6, 11, and 14 could, to a large extent (although not completely), be explained by known protein variants (of $\beta$-casein, $\kappa$-casein, or $\beta$-lactoglobulin) or the previously described DGAT1 K232A polymorphism. Additionally, 27 regions distributed across 18 chromosomes showed smaller effects on individual milk proteins. These results provide important new insights in genetic aspects of milk protein composition.

Risk factors associated with selected indicators of milk quality in semi-arid northeastern Brazil. By Oliveira et al., page 3166. This study was performed to gain information on quality traits of bulk milk produced by small- and medium-scale producers in a semi-arid northeastern region of Brazil. Although the majority of the farms are in accordance with the current regulations for total bacteria $(88 \%)$ and somatic cell counts (94\%), nearly half of the producers $(46 \%)$ would have problems in achieving the planned (2012) threshold limit for total bacteria count if no improvement in milk quality occurs. Premilking teat-end wash procedure and postmilking teat dip were associated with lower total bacteria and Staphylococcus aureus counts in bulk milk, respectively.

When cheese gets the blues: Pseudomonas fluorescens as the causative agent of cheese spoilage. By Martin et al., page 3176. A fresh, Latin-style cheese was contaminated with Pseudomonas fluorescens, which produced a blue fluorescent pigment on the surface of the cheese. A variety of classical and molecular microbiological methods were used to identify the causative microbe and track contamination in the processing environment. A combination of improved cleaning and sanitation procedures in the processing facility and the addition of starter cultures and potassium sorbate to the cheese making procedures ultimately eliminated the contamination.

Maximizing profit on New England organic dairy farms: An economic comparison of 4 total mixed rations for organic Holsteins and Jerseys. By Marston et al., page 3184. Because feed costs are generally the highest input cost on organic dairy operations, large quantities of homegrown feed make up the bulk of organic dairy rations. Meeting the nutritional requirements of lactating organic dairy cows using high forage rations can be challenging; therefore; studies that compare production parameters and the economic efficiency of rations for organic dairy cows are necessary. Feeding a grass silage-based diet supplemented with commodity concentrates may provide the greatest 
economic return for New England organic dairy producers.

The genomic evaluation system in the United States: Past, present, future. By Wiggans et al., page 3202. The implementation of genomic evaluation for dairy cattle has caused profound changes in dairy cattle breeding. The reliability of those evaluations can reach around $75 \%$ for yield traits. This technology, which resulted from an international consortium of government, university, and industry cooperators, became available in December 2007, and the first unofficial USDA evaluations based on single nucleotide polymorphism genotypes were released in April 2008. A steady increase in evaluation accuracy has resulted from including additional bulls with genotypes and traditional evaluations. Exchange of genotypes with other countries has increased the size of data sets to give more accurate genomic evaluations, and collaborations are expected to increase in the future. 